\title{
Segregation of isoenzyme markers and meiotic pairing control genes in Lolium
}

\section{Taing Aung and}

G. M. Evans
Department of Agricultural Botany, University

College of Wales, Aberystwyth, Dyfed, U.K.

The genetic basis of the control system responsible for suppressing the association of homoeologous chromosomes at first metaphase of meiosis in interspecific hybrids of Lolium was investigated by means of cytological and genetical analyses of a test cross between $L$. temulentum and $L$. perenne. It is concluded that more than one gene is involved. Linkage to a gene which codes for an Esterase enzyme of low electrophoretic mobility is clearly identified with the possibility that a second gene is associated with another Esterase locus. The phenotypic effect of individual pairing genes in relation to the distance between them and linked markers is discussed.

\section{INTRODUCTION}

All species of the genus Lolium are diploids with $2 n=2 x=14$. Chromosome pairing in hybrids between the various species is extensive with seven bivalents being the norm at metaphase one of meiosis. This occurs even in hybrids between the inbreeder $L$. temulentum and the outbreeder $L$. perenne despite there being a difference of 50 per cent in chromosome size and DNA content between the two species (Hutchinson, Rees and Seal, 1979). However, some genotypes of $L$. perenne are known to carry genetic factors which suppress homoeologous chromosome association in both diploid and polyploid interspecific hybrids (Evans and Macefield, 1972; Taylor and Evans, 1976; 1977; Taing Aung and Evans, 1985; Evans and Davies, 1985). These factors are located both in the normal $A$ chromosome complement and in supernumary $B$ chromosomes.

This paper is a report of a preliminary investigation into the distribution of these 'pairing control' genes within the $L$. perenne genome. The method used was to measure chromosome pairing in terms of the frequency of chiasmata and univalents at first metaphase of meiosis in the progeny of a cross between $L$. perenne and $L$. temulentum and to test for any correlation between the level of pairing and the segregation of isoenzyme marker genes i.e., to test for linkage between the level of pairing and marker genes. In an assay of this type, not only must the $L$. perenne parent be heterozygous for the 'pairing control' gene(s), it must also be heterozygous for as many isoenzyme genes as possible. In this assay we only had three such markers available in the $L$. perenne parent.

\section{MATERIALS AND METHODS}

\section{Stocks}

The L. perenne parent used was Lp18. It was derived from a cross between the 'high pairing' genotype Lp10 and the 'low pairing' genotype Lp19. Although details of the origin and properties of these two genotypes have been given by Taylor and Evans (1977) and by Evans and Davies (1985), it is worth pointing out that they are half sibs derived from a cross between a single heterozygous $L$. perenne plant of Algerian origin and a pollen cloud from a commercial cultivar named $S 24$. Evidence was available to indicate that Lp18 was heterozygous for genes controlling chromosome pairing at meiosis and for a phosphoglucoisomerase (Pgi-2) and two esterase (Est.) genes. There were no $B$ chromosomes present in this plant.

The L. temulentum parent used for the test cross was $\mathrm{Ba} 3081$. This is a long established pure line which has been used several times to identify the presence of 'pairing control' factors in Lolium (Evans and Macefield, 1972; Taylor and Evans, 
1976; 1977; Evans and Davies, 1985; Evans and Taing Aung, 1985).

\section{Test cross}

Emasculated inflorescences of $\mathrm{Ba} 3081$ were hand pollinated with pollen from Lp18. Since the endosperm does not develop properly in the hybrid seed, 20 day embryos were dissected from the caryopses and cultured on a suitable culture medium (Gamborg, 1970). The hybrid seedlings were transplanted into soil at the three leaf stage.

\section{Isoenzyme assay}

One hundred and thirty nine hybrid progeny together with the parents, Ba 3081 and Lp18, were assayed to determine the individual genotypes with respect to three segregating enzyme loci. These were Pgi-2 (phosphoglucoisomerase) and two Esterase loci which we have labelled here as Est-a and Est-c. These were identified by electrophoretic bands nearest the anodal front and the cathodal origin respectively. The genetic control of the many Esterase isoenzyme bands found in Lolium has not been clearly defined and consequently we feel it would be inappropriate to allocate numbers to these loci at the present time. Est-a would presumably correspond to Est-1 in other genera. We do however have sufficient information to indicate that both Est-a and Est-c genes are represented in this material by an allele $(+)$ which gives a clear dark staining band and a "null" (0) allele which gives no band.

\section{Electrophoresis}

Both the Pgi and the Esterase enzymes were separated from a crude extract by horizontal starch electrophoresis. Young leaves were crushed in a few drops of $0 \cdot 1 \mathrm{M}$ Tris mercaptoethanol buffer at $7 \cdot 2$ and the liquid absorbed into small paper wicks and placed in a transverse cut in the starch gel in the usual way. Electrophoresis was conducted at $4^{\circ} \mathrm{C}$. at 250 volts and using $0.2 \mathrm{M}$ Lithium borate pH 8.3 as the electrode buffer. The paper wicks were removed after approximately 30 minutes and the run continued for a further 4-5 hours. On completion, the gel was split horizontally into two with one slice being stained for Pgi and the second for Est. The staining solutions were as follows.

\section{Pgi}

$15 \mathrm{mg} \quad$ Fructose-6-phosphate, $15 \mathrm{mg}$ MTT, $5 \mathrm{mg}$ Phenazine

$8 \mathrm{mg}$ NADP, methosulphate
(PMS), 20 units Glucose-6-dehydrogenase $1 \mathrm{ml}$ $1 \mathrm{M}$ Magnesium chloride, $100 \mathrm{ml} 0 \cdot 1 \mathrm{M}$ Tris $\mathrm{HCl}$ $p \mathrm{H} 7 \cdot 5$. Incubation was in the dark at $37^{\circ} \mathrm{C}$.

\section{Est}

$2 \mathrm{ml} 1$ per cent $a$-napthyl acetate (in acetone), $1 \mathrm{ml} B$-napthyl acetate (in acetone), $100 \mathrm{mg}$ Fast blue RR salt, $100 \mathrm{ml} 0 \cdot 1 \mathrm{M}$ Phosphate buffer $p \mathrm{H} 7 \cdot 0$. Incubation was in the dark at $37^{\circ} \mathrm{C}$. Gels were fixed in 50 per cent glycerol and the position of each stained isoenzyme band recorded.

\section{Cytological analysis}

Emerging inflorescences were fixed in Carnoy's fixative and squash preparations of pollen mother cells $(\mathrm{pmc})$ at first metaphase of meiosis made in acetocarmine stain. The frequencies of univalents and chiasmata in 20 pmc per hybrid plant and in $50 \mathrm{pmc}$ for each of the parents were subsequently recorded and the mean values calculated.

\section{RESULTS}

\section{Cytological analysis}

A total of 125 out of the 139 hybrid plants were scored. Suppression of homoeologous association at first metaphase is reflected by a high univalent frequency and a low chiasma frequency and vice versa. In fact the univalent frequency alone is in most instances a more accurate reflection of the level of pairing than is chiasma frequency.

Chromosome pairing in both parents was complete with no univalents being recorded in any of the $50 \mathrm{pmc}$ scored in the single Lp18 and in a number of $\mathrm{Ba} 3081$ plants. However the pattern of homoeologous association in the test cross progeny ranged from complete pairing (no univalents) to relatively low pairing ( 7.9 univalents). This was reflected also in the mean pmc chiasma frequency per plant which ranged from $12 \cdot 85$ to $4 \cdot 35$. Moreover there was no obvious groupings into distinct classes. This is shown diagramatically in fig. 1 where the distribution of mean univalent and mean chiasma values are plotted. Although an incremental value of one was arbitrarily chosen for each distribution a change to 0.5 or to 1.5 does not change the pattern of the final histogram. The absence of two distinct peaks is of course indicative of a genetic system involving more than one gene. 


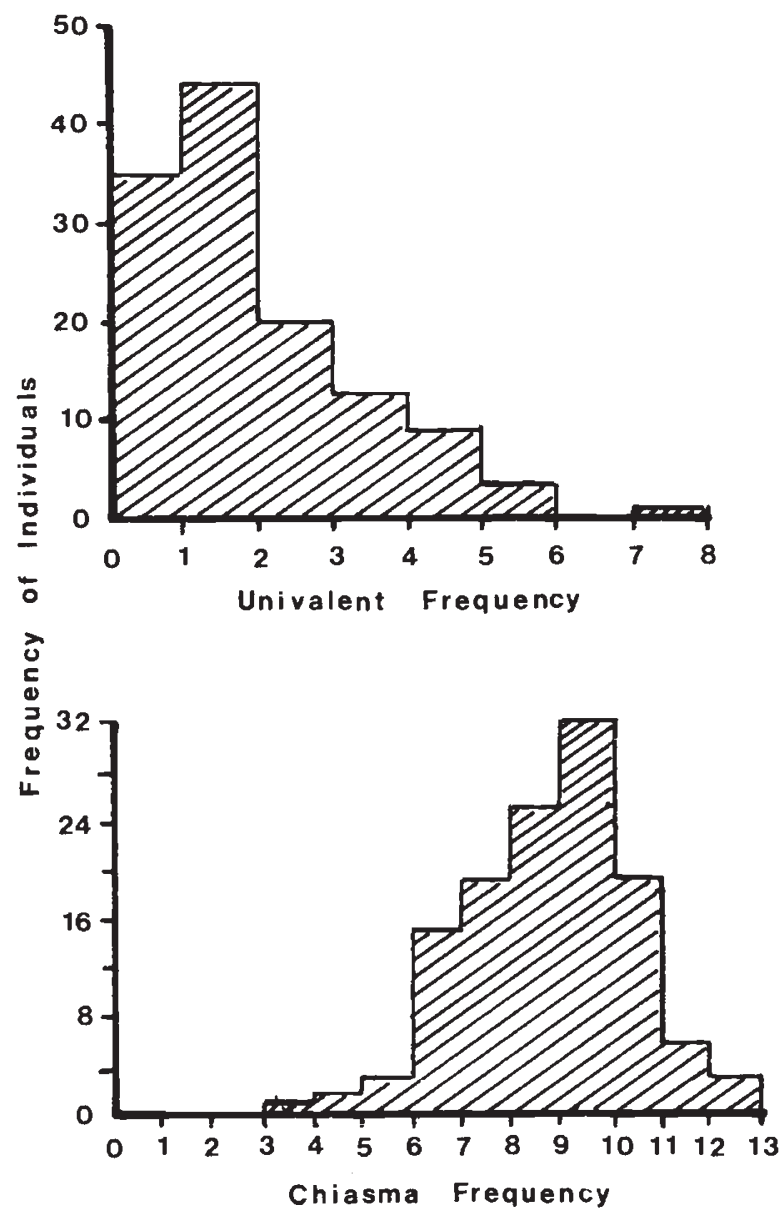

Figure 1 Frequency distributions of mean numbers of univalents and of chiasmata per cell per plant of the test cross progeny.

\section{Isoenzyme assay}

\section{Parents}

L. temulentum $\mathrm{Ba} 3081$ is known to be highly inbred and no isoenzyme heterozygosity or polymorphism has been found in the present stock. It is homozygous for the $b$ allele of Pgi-2 and since it gives no band for both Esterases we consider it to be homozygous for a null allele for both Est-a and Est-c.

The $L$. perenne $\mathrm{Lp} 18$ is heterozygous at all three loci. It gives the classical three banded $a b$ phenotype for $P g i-2$ and a single dark staining band at both the anodal and cathodal extremes of the Esterase gel. From the results of the test cross which are given below we interpret the genotype of Lp18 as being Est-a+/0 and Est-c $+/ 0$.

\section{Progeny}

The progeny segregated into two distinct phenotypic groups for each of the three enzyme systems. This was a direct consequence of the heterozygous nature of all three loci in the Lp18 parent. The phenotypes of the parents and of the eight segregant classes together with the frequency of progeny in each of the classes are given in fig. 2 .

The data were analysed for monogenic segregation at the three loci and also for linkage between any two using a simple $\chi$-square test (table 1 ). No significant deviation from the expected $1: 1$ ratio of homozygotes to heterozygotes was detected for any of the loci although the $\chi$-square value for the Est- $a$ gene was quite substantial. The analysis also shows no indication of linkage between any of the three loci.

Table 1 Evaluation of the segregation of the three enzyme genes

\begin{tabular}{llll}
\hline Locus & Frequencies & $\mathrm{N}$ & $\chi_{(1)}^{2}(1: 1)$ \\
\hline Pgi-2 $(a-b)$ & $70-69$ & 139 & $0 \cdot 007$ \\
Est $-a(+-0)$ & $79-60$ & 139 & $2 \cdot 597$ \\
Est $-c(+-0)$ & $78-61$ & 139 & $2 \cdot 079$ \\
Linkage & & & \\
Pgi-2/Est $-a$ & $68-71$ & 139 & 0.065 \\
Pgi-2/Est $-c$ & $61-78$ & 139 & $2 \cdot 079$ \\
Est $-a /$ Est $-c$ & $68-71$ & 139 & 0.065 \\
Pgi-2/Est $-a /$ Est $-c$ & $69-70$ & 139 & 0.007 \\
\hline
\end{tabular}

\section{Analysis of the segregation of isoenzyme markers and pairing pattern at metaphase one of meiosis in the test cross progeny}

In an attempt to detect linkage between any of the marker enzyme genes and the genetic factors which determine the degree of chromosome association at first metaphase of meiosis in these interspecific hybrids, the progeny were divided into the eight segregant classes based on their isoenzyme profile for the three enzyme systems. The mean number of chiasmata and univalents for the eight classes is given in table 2 and the analysis of variance of the data given in table 3 . The variation between plants within classes is used as the estimate of error variance for estimation of the variance ratio. As with many analysis of this type the number of individuals falling into each class differs and consequently the orthogonal partitioning of the results into effects of allelic variation at each locus has to be done on the class means with adjustments of the within class variation using the harmonic mean. This analysis forms the second part of table 3 . A 


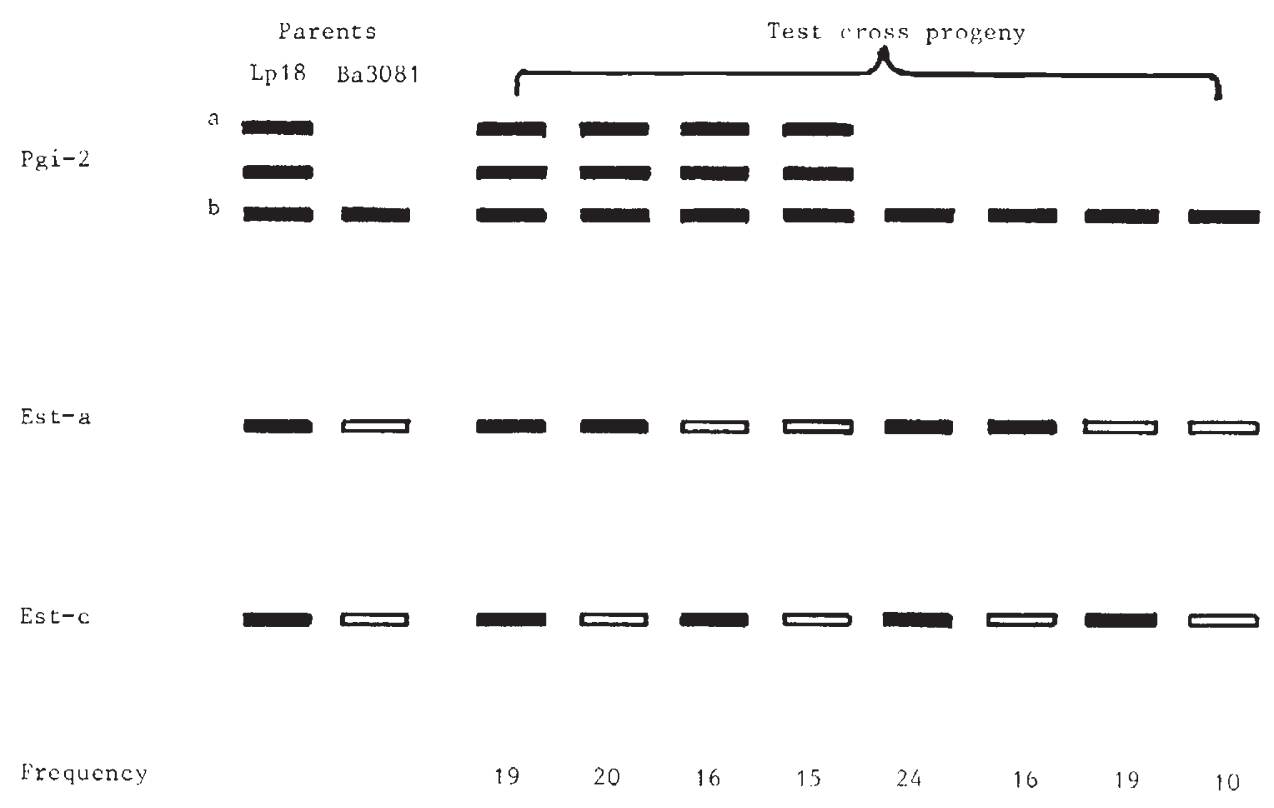

Figure 2 Isoenzyme banding phenotypes for Pgi-2, Est-a and Est-c of the two parents and the eight classes of progeny together with the number of plants in each of the segregant classes. $(\square=$ null).

significant difference between the segregant classes of any of the three marker genes is taken as evidence of linkage between that gene and gene or genes controlling the degree of homoeologous association at metaphase one of meiosis. On this basis both Esterase loci seem to be linked to "pairing control" genes although the difference between the two segregant classes of Est-a is only significant at the 5 per cent level. The difference between the $P g i-2$ segregants was not significant. As might, be expected, bearing in mind that there is no linkage between Pgi-2 and pairing genes and that the evidence of linkage between Est- $a$ and pairing is not convincing, the "interaction" components in the analysis are very small. They are included as one item with four degrees of freedom in (table 3) although they were initially analysed separately.

\section{Linkage to Est-c}

If an isoenzyme marker is linked to a pairing control gene the difference in pairing (univalents/bivalents/chiasma frequency) observed between the two segregant classes of the marker will be the product of the effect of the pairing gene and the percentage recombination (distance)

Table 2 Genotypes of the parents and of the 8 classes of progeny together with the mean number of chiasmata and univalents for each class

\begin{tabular}{|c|c|c|c|c|c|c|}
\hline & \multicolumn{3}{|c|}{ Genotypes } & \multirow[b]{2}{*}{$N$} & \multicolumn{2}{|c|}{ Mean number of } \\
\hline & Pgi-2 & Est-a & Est-c & & Chiasmata & Univalents \\
\hline \multicolumn{7}{|l|}{ Parents } \\
\hline Lp18 & $a / b$ & +10 & $+/ 0$ & & & \\
\hline Ba3081 & $b / b$ & $0 / 0$ & $0 / 0$ & & & \\
\hline \multicolumn{7}{|l|}{ Progeny } \\
\hline & $a / b$ & $+/ 0$ & $+/ 0$ & 17 & $9 \cdot 082$ & 1.659 \\
\hline & $a / b$ & $+/ 0$ & $0 / 0$ & 18 & $9 \cdot 611$ & $1 \cdot 172$ \\
\hline & $a / b$ & $0 / 0$ & $+/ 0$ & 13 & $8 \cdot 212$ & $2 \cdot 223$ \\
\hline & $a / b$ & $0 / 0$ & $0 / 0$ & 10 & 8.915 & 1.660 \\
\hline & $b / b$ & $+/ 0$ & $+/ 0$ & 24 & $8 \cdot 340$ & $2 \cdot 283$ \\
\hline & $b / b$ & $+/ 0$ & $0 / 0$ & 15 & $9 \cdot 550$ & $1 \cdot 393$ \\
\hline & $b / b$ & $0 / 0$ & +10 & 18 & $7 \cdot 743$ & 2.778 \\
\hline & $b / b$ & $0 / 0$ & $0 / 0$ & 10 & 8.675 & 1.970 \\
\hline
\end{tabular}


Table 3 Analysis of variance of the differences in chiasma and univalent frequencies between the segregant classes of the three enzyme loci.

\begin{tabular}{|c|c|c|c|}
\hline & DF & Chiasmata & Univalents \\
\hline Total & 124 & & \\
\hline Between classes & 7 & $7 \cdot 3227^{*}$ & $4 \cdot 8132^{*}$ \\
\hline Within classes & 117 & $2 \cdot 7449$ & $1 \cdot 8769$ \\
\hline \multicolumn{4}{|l|}{$\begin{array}{l}\text { Orthogonal partitioning } \\
\text { of between class means }\end{array}$} \\
\hline$P g i-2(a b$ v. $b b)$ & 1 & 0.2865 & $0 \cdot 3655$ \\
\hline Est-a $(+0$ v. 00$)$ & 1 & $1 \cdot 1551^{*}$ & $0.5639^{*}$ \\
\hline Est-c $(+0$ v. 00$)$ & 1 & $1 \cdot 4248^{* *}$ & $0.9439 * *$ \\
\hline Residual (Interactions) & 4 & 0.0329 & 0.0139 \\
\hline $\begin{array}{l}\text { Within classes } \\
\text { (Adjusted for means) }\end{array}$ & 117 & $0 \cdot 1905$ & $0 \cdot 1303$ \\
\hline
\end{tabular}

** $P=<0.01 ; * P=<0.05$.

between it and the marker. This assumes that a single gene is being assayed and that any other pairing control genes segregate independently of the one being assayed and that the sample is large enough for the 'background effect' to equalise over the two segregant classes of the marker. If the effect on pairing (univalent frequency) of the gene being assayed is denoted as $P h$, the Recombination fraction (= Recombination frequency) as $R f$ and the effect of the other pairing genes plus environmental effects (background effect) as $B$, then the frequency of univalents expected from the two segregating classes of the linked marker will be $B+P h-$ $(P h \times R f)$ and $B+(P h \times R F)$ respectively. The mean difference $(\bar{D})$ in univalent frequency between the two classes would then be $B+P h-$ $(P h \times R f)-(B+P h \times R f))$ which can be reduced to $\bar{D}=P h(1-2 R F)$. Only $\bar{D}$ can be obtained with any precision from the assay described in the previous section. The overall mean difference in univalents between the two segregating classes of Est-c was $0 \cdot 77$. This still leaves $P h$ and $R f$ as unknowns in the equation and without another linked marker it is impossible to obtain an accurate measure of $R f$ and consequently of $P h$.

However a crude estimate of $P h$ is given by the difference between the maximum values of univalents per plant recorded in the two segregant classes of Est-c. This assumes that the difference in pairing between the two plants is due entirely to the different effect of the two alleles of the gene linked to Est-c and that one of the alleles has no effect on homoelogous pairing. The probability that the maximum effect of the other gene(s) affecting pairing is present in both plants depends on the number of genes involved in relation to the size of the sample. The maximum number of uni- valents per plant recorded in the $+/ 0$ class was 7.9 compared with a value of 5.5 in the $0 / 0$ segregants. This gives a phenotypic effect of $2 \cdot 4$ univalents attributable to the pairing gene linked to Est-c. It is emphasised however, that this must be treated at as an approximate estimate only. With a $P h$ value of this magnitude the distance between the pairing gene and the Est-c marker would be quite substantial (an $R f$ value of $0 \cdot 34$ ).

The estimate is that $L$. perenne Lp18 carries a pairing control allele placed approximately 34 map units from the Est-c locus and which is capable of reducing the degree of pairing in interspecific hybrids with $L$. temulentum to such an extent as to increase the number of univalents by approximately $2 \cdot 4$ per cell.

\section{DISCUSSION}

Taylor and Evans (1977) and Evans and Davies (1985) have clearly established that some genotypes of $L$. perenne have a genetic system which is capable of suppressing homoelogous pairing at meiosis in hybrids with $L$. temulentum. The present report is an attempt to describe the genetic basis of this variation in a little more detail.

The evidence from the orthodox cytological analysis of metaphase one of meiosis in hybrids and from the genetic assay for linkage of pairing pattern to isoenzyme genes suggest that more than one gene is involved. The cytological analysis of 125 progeny failed to identify distinct groups of individuals in terms of the pattern of chromosome association at first metaphase of meiosis. This still does not rule out the possibility that relatively few genes are involved and that one or two could have the major effect with others having a much more minor role.

The evidence that a pairing control gene is linked to Est-c is strong. That pairing control is linked to Est-a is more questionable although still possible. The evidence is based entirely on the level of difference in univalent and chiasma frequency between the two segregant classes of the enzyme system. A marginal level of significance for the differences between classes of this type could be due to one or more of the following reasons. Firstly, there is the possibility that there is no linkage between the marker and pairing and that the difference obtained is spurious due to ineffective or inadequate sampling. Secondly, linkage could be present but the map distance between the two loci is large. Thirdly, there is the possibility that the effect on pairing of the gene being mapped is 


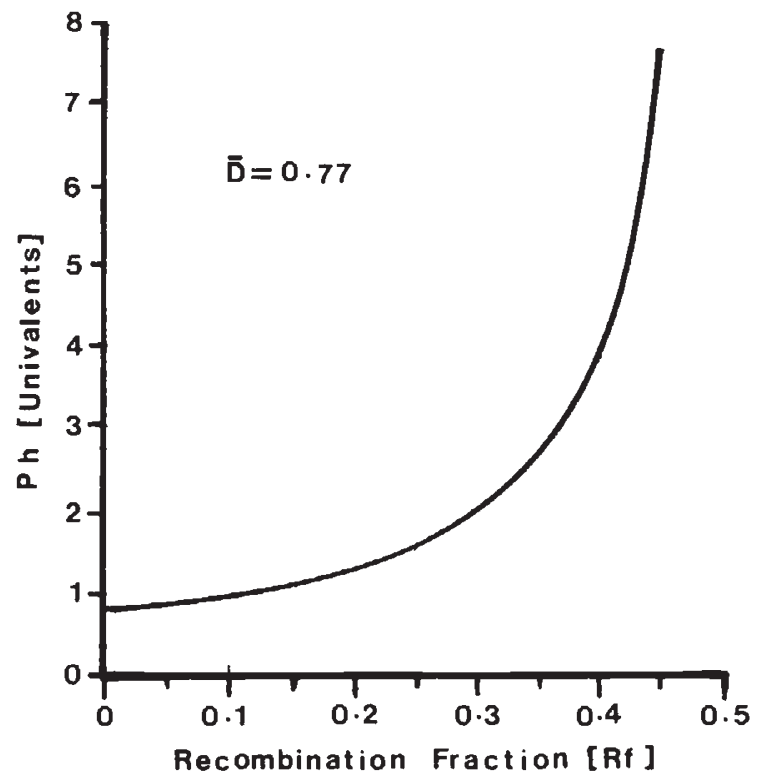

Figure 3 Relationship between phenotypic effect $(P h)$ measured as univalents per cell of a pairing gene and the recombination fraction $(R f)$ between it and a linked marker using a fixed value of mean difference $(\bar{D})$ between the segregant classes of the marker of 0.77 univalents.

small and even if linkage between it and the marker is relatively close the difference between the two segregating classes of progeny would be difficult to detect with any level of confidence.

Sample size in this assay is not large and it could be argued that a difference between classes of only 5 per cent significance as in the case of Est-a is suspect.

Even if this suspect linkage is disregarded the information still points to there being more than one pairing gene segregating in this material. The estimate of the effect of the pairing gene linked to Est-c cannot possibly account for the full range of pairing pattern observed in the test cross progeny. The crude estimate of the phenotypic effect of this gene is to suppress homeologous pairing to the extent that approximately 2.4 univalents are produced. Although substantial it is still only about 30 per cent of the maximum number of 7.9 univalents observed in the 125 hybrids examined.

It was shown in the previous section that the phenotypic effect $(P h)$ of a gene being assayed, its distance from a linked marker (Recombination fraction) and the mean phenotypic difference $(\bar{D})$ between the two segregating classes of the marker are directly related to the extent that $\bar{D}=$ $P h(1-2 R f)$. It follows therefore that a given value of $\bar{D}$ could be achieved at the one extreme by a value of $P h$ very similar to that of $\bar{D}$ allied to tight linkage to the marker or at the other extreme by a high value of $P h$ with a high $R f$ value. For example if $R f=0$, i.e., complete linkage of the marker and the pairing gene, the value of $\mathrm{Ph}$ will equal that of $\bar{D}$ and, in contrast, if $R f=0.45$ then $P h$ will assume a value of ten times that of $\bar{D}$.

A plot of the relationship between $P h$ and $R f$ for a $\bar{D}$ value of 0.77 (that between the two Est-c segregant classes) is given in fig. 3. This relationship between Recombination fraction and the effect of a pairing gene in producing a specific difference between the segregating classes of a marker gene is however not linear. There is a disproportionate increase in the value of $P h$ with increasing values of recombination. It follows that the error attached to estimates of the phenotypic effect of individual genes increases disproportionately as the distance between it and its linked marker increases. It is stressed again that the value of 2.4 univalents given above as the effect produced by the gene linked to Est-c must be treated as very approximate bearing in mind that an accurate estimate of $\mathrm{Rf}$ is not possible.

The method described here of partitioning the genetic basis of the variation in homoeologous chromosome pairing at meiosis in hybrids can be developed further with the identification of new isoenzyme markers. It is appreciated however that the present model is based on additive gene action only and it would be surprising if this would account for all the variation observed.

Acknowledgements We are grateful to the AFRC for financial support and to $\mathrm{Mr}$ D. Fallding for his expert technical assistance.

\section{REFERENCES}

EVANS, G. M. AND DAVIES, E. W. 1985. The genetics of meiotic chromosome pairing in Lolium temulentum $\times$ Lolium perenne tetraploids. Theor. Appl. Genet., 71, 185-192.

EVANS, G. M. AND MACEFIELD, A. J. 1972. Suppression of homoeologous pairing by B chromosomes in a Lolium species hybrid. Nature N.B., 236, 110-111.

HUTCHINSON, $y$, REES, H. AND SEAL, A. G. 1979. An assay of the activity of supplementary DNA in Lolium. Heredity, 43, 411-421.

(iAMBORG, O. I. 1970. The effects of amino acids and ammonium on growth of plant cells in suspension culture. Plant Physiol., 45, 372-375.

TAING AUNG ANI) EVANS, G. M. 1985. The potential for diploidizing Lolium multiflorum $\times$ L. perenne tetraploids. Can. J. Genet. Cytol., 27, 506-509.

TAYLOR, 1. B. AND EVANS, (i. M. 1976. the effect of B chromosomes on homoeologous pairing in species hybrids. III. Intraspecific variation. Chromosoma, 57, 25-32.

TAYLOR, 1. B. AND EVANS, G. M. 1977. The genetic control of homoeologous chromosome association in Lolium temulentum $\times$ L. perenne. Chromosoma, 62, 57-67. 